\title{
Optimization under Generalized Intuitionistic Fuzzy Environment
}

\author{
Arindam Garai* \\ Sonarpur Mahavidyalaya \\ South 24 Parganas \\ West Bengal, India, Pin 700149
}

\author{
Tapan Kumar Ray \\ Bengal Engineering and Science University \\ Shibpur, Howrah \\ West Bengal, India, Pin: 711103
}

\begin{abstract}
This paper presents the concept of generalized intuitionistic fuzzy set (GIFS) and optimization technique under generalized intuitionistic fuzzy environment. The idea of GIFS was introduced by Tapas K. Modal et al. Here, another new GIFS has been introduced. Solution technique of optimization problem involving both types of GIFS has been discussed. For the sake of simplicity alone, the same problem, as studied by Angelov, who first well developed the intuitionistic fuzzy optimization technique in 1997, is taken. Conclusions are obtained finally.
\end{abstract}

\section{General Terms}

Fuzzy optimization, generalized intuitionistic fuzzy set.

\section{Keywords}

Type I generalized intuitionistic fuzzy set, type II generalized intuitionistic fuzzy set, intuitionistic fuzzy optimization, generalized intuitionistic fuzzy optimization.

\section{INTRODUCTION}

In over the last three decades, optimization problems have been investigated in the sense of fuzzy set theory [8]. Fuzzy optimization [4] formulations are much flexible and these allow finding solutions that are more adequate to the real problem in comparison with crisp problems

Again, fuzzy set theory [8] has been developed in detail and various modifications and generalizations have appeared. One of them is the concept of intuitionistic fuzzy (IF) sets [2, 3]. These consider not only the degree of membership to a given set, but also the degree of rejection so that the sum of both the values is less than or equal to one. Applying this concept Plamen Angelov [1] had reformulated the optimization problem.

Recently Tapas Mondal et al. has defined generalized IF set [7]. Yet, optimization under generalized IF environment has not been considered yet. In this paper, optimization problem under generalized intuitionistic fuzzy environment has been considered and solved.

Angelov [1] and many other researchers have well identified that, in general, an optimization problem includes objective(s) and constraint(s). In fuzzy optimization problems, the objective(s) and/or constraint(s) or parameter(s) and relation(s) are described by fuzzy sets. The solution of crisp optimization problem must satisfy all the constraints exactly where as in the case of analogous fuzzy optimization problem; the degree of satisfaction of objective(s) and of constraint(s) is maximized. It is then reformed via Bellman-Zadeh's approach [4]. When the degree of rejection (non-membership) is defined along with the degree of acceptance (membership) as

well as when both these degrees are not complementary to each other, IF sets can be used as a more general and full tool for describing this uncertainty [5].

Similarly, in case of generalized intuitionistic fuzzy sets, degree of acceptance is maximized and the degree of rejection is minimized while keeping in mind the concurrent restriction.

In this paper, at first, the definitions of fuzzy and intuitionistic fuzzy set are given. Next, definitions of two types of generalized intuitionistic fuzzy set (GIFS) have been introduced. Next, the idea of fuzzy and intuitionistic fuzzy optimization has been revisited in brief. Next, optimization under GIFS (for both type I and type II GIFS) has been discussed in detail. Finally an example has been taken and conclusions are discussed.

\section{DEFINITION}

\subsection{Fuzzy Set}

A fuzzy subset [8] $\tilde{A}$ of $X$ is defined by its membership function $\mu_{\tilde{\mathrm{A}}}: X \rightarrow[0,1]$ that assigns to every $\mathrm{x} \in \mathrm{X}$, a real number $\mu_{\tilde{\mathrm{A}}}(\mathrm{x})$ in the closed unit interval $[0,1]$, where the value of $\mu_{\tilde{\mathrm{A}}}(\mathrm{x})$ at $\mathrm{x}$ represents the grade of membership of $\mathrm{x}$ in $\tilde{A}$.

Nearer the value of $\mu_{\tilde{\AA}}(\mathrm{x})$ is unity, the grade of membership of $x$ in $\tilde{A}$. When the membership function $\mu_{\tilde{A}}(\mathrm{x})$ contains only two points 0 and 1 , membership function $\mu_{\tilde{\mathrm{A}}}(\mathrm{x})$ is identical to the characteristic function $\chi: \mathrm{X} \rightarrow[0,1]$ and in that case $\tilde{\mathrm{A}}$ is a crisp set.

\subsection{Intuitionistic Fuzzy Set}

An intuitionistic fuzzy set [3] $\mathrm{A}$ in $\mathrm{X}$ is defined by $\mathrm{A}=\{<\mathrm{x}$; $\left.\mu_{\mathrm{A}}(\mathrm{x}), v_{\mathrm{A}}(\mathrm{x})>\mid \mathrm{x} \in \mathrm{X}\right\}$, where $\mu_{\mathrm{A}}: \mathrm{X} \rightarrow[0,1]$ and $v_{\mathrm{A}}: \mathrm{X} \rightarrow[0$, 1] with the constraint $0 \leq\left(\mu_{\mathrm{A}}(\mathrm{x})+v_{\mathrm{A}}(\mathrm{x})\right) \leq 1$, where $\mu_{\mathrm{A}}(\mathrm{x})$ and $v_{\mathrm{A}}(\mathrm{x})$ denote the degree of membership and non membership respectively.

\subsection{Generalized Intuitionistic Fuzzy Set}

Atanassov introduced the concept of intuitionism in fuzzy set theory. Degree of membership of an element in a set in considered in fuzzy set theory where as in case of intuitionistic fuzzy set theory $[3,6]$, degree of non membership is also considered. It was assumed by Atanassov that the degree of membership and non membership do not overlap so that their sum must be less than or equal to one. But, degree of acceptance and degree of rejection may overlap in some cases. $[9,10]$ In that case, ideas of generalized intuitionistic fuzzy set of different types come out.

\section{$\mu$}

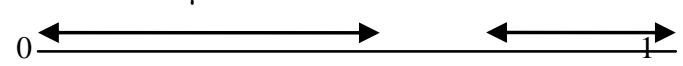


Fig 1: Atanassov's intuitionistic fuzzy set

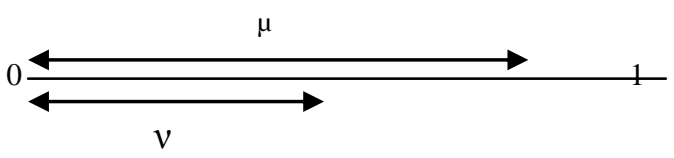

Fig 1: Generalized intuitionistic fuzzy set

\subsubsection{Type I Generalized IF Set}

A type I generalized intuitionistic fuzzy subset [7] A of universal set $\mathrm{X}$ is an object of the form $\mathrm{A}=\left\{<\mathrm{x} ; \mu_{\mathrm{A}}(\mathrm{x}), v_{\mathrm{A}}(\mathrm{x})\right.$ $>\mid \mathrm{x} \in \mathrm{X}\}$ where the functions $\mu_{\mathrm{A}}: \mathrm{X} \rightarrow[0,1]$ and $v_{\mathrm{A}}: \mathrm{X} \rightarrow$ $[0,1]$ with the constraint: $\min \left(\mu_{\mathrm{A}}(\mathrm{x}), v_{\mathrm{A}}(\mathrm{x})\right) \leq 0.5$.

Clearly, $0 \leq \min \left(\mu_{\mathrm{A}}(\mathrm{x}), v_{\mathrm{A}}(\mathrm{x})\right) \leq 0.5$. It is to be noted that all the IFSs are type I generalized intuitionistic fuzzy set but the converse does not hold good.

\subsubsection{Type II Generalized IF Set}

A type II generalized intuitionistic fuzzy subset $\mathrm{A}$ of universal set $\mathrm{X}$ is an object of the form $\left.\mathrm{A}=\left\{<\mathrm{x} ; \mu_{\mathrm{A}}(\mathrm{x}), v_{\mathrm{A}}(\mathrm{x})\right\rangle \mid \mathrm{x} \in \mathrm{X}\right\}$ where the functions $\mu_{\mathrm{A}}: X \rightarrow[0,1]$ and $v_{\mathrm{A}}: \mathrm{X} \rightarrow[0,1]$ with the condition $\left(\mu_{\mathrm{A}}(\mathrm{x}) * v_{\mathrm{A}}(\mathrm{x})\right) \leq 0.25$.

Clearly, $0 \leq\left(\mu_{\mathrm{A}}(\mathrm{x}) * v_{\mathrm{A}}(\mathrm{x})\right) \leq 0.25$. It is to be noted that all the IFSs are type II generalized intuitionistic fuzzy set but the converse does not hold good.

It is also noted that all type II GIFS are type I GIFS but the converse is not true in general. It is well known that if $0 \leq a$, $\mathrm{b} \leq 1, \mathrm{a} \cdot \mathrm{b} \leq \mathrm{a}$ and $\mathrm{a} \cdot \mathrm{b} \leq \mathrm{b}$. Then $\mathrm{a} \cdot \mathrm{b} \leq \min (\mathrm{a}, \mathrm{b})$. Hence $\left(\mu_{\mathrm{A}}(\mathrm{x})\right.$ $\left.* v_{\mathrm{A}}(\mathrm{x})\right) \leq \min \left(\mu_{\mathrm{A}}(\mathrm{x}), v_{\mathrm{A}}(\mathrm{x})\right)$

\section{OPTMIZATION TECHNIQUE \\ 3.1 Crisp, Fuzzy and IF Optimization}

\subsubsection{Crisp Optimization}

A crisp optimization problem of the form

$\begin{array}{lll}\text { Minimize } & f_{i}(x), & i=1 \ldots p, \\ \text { Subject to the constraints } & \\ g_{j}(x) \leq 0 & j=1 \ldots q .\end{array}$

$$
\mathrm{g}_{\mathrm{j}}(\mathrm{x}) \leq 0 \quad \mathrm{j}=1 \ldots \mathrm{q} .
$$

Where $x$ denotes the unknown variables, $f_{i}(x)$ denotes the objective functions, $\mathrm{g}_{\mathrm{j}}(\mathrm{x})$ denotes the constraints (nonequalities), $p$ denotes the number of objectives and $q$ denotes the number of constraints. The solution of this crisp optimization problem satisfies all constraints exactly.

\subsubsection{Fuzzy Optimization}

In the analogous fuzzy optimization problem, the degree of satisfaction of objective(s) as well as of constraint(s) is(are) maximized.

The problem takes the form:

Minimize $\quad \mathrm{f}_{\mathrm{i}}(\mathrm{x}), \quad \mathrm{i}=1 \ldots \mathrm{p}$,

Subject to the constraints

$$
\mathrm{g}_{\mathrm{j}}(\mathrm{x}) \stackrel{\sim}{\leq} 0, \quad \mathrm{j}=1 \ldots \mathrm{q} .
$$

Where Minimize denotes the fuzzy minimization and $\tilde{\leq}$ denotes the fuzzy inequality.

Next, it is transformed via Bellman-Zadeh's approach to the following optimization problem: to maximize the degree of membership (acceptance) of the objective(s) as well as of the constraints to the respective fuzzy sets:

Maximize $\quad \mu_{\mathrm{i}}(\mathrm{x}), \quad x \in R^{n}, i=1 \ldots(p+q)$

Subject to the constraints

$$
0 \leq \mu_{\mathrm{i}}(\mathrm{x}) \leq 1 \text {. }
$$

Where $\mu_{\mathrm{i}}(\mathrm{x})$ denotes degree of acceptance of $\mathrm{x}$ in $\boldsymbol{R}^{n}$.

\subsubsection{Intuitionistic Fuzzy Optimization}

To solve the optimization problem under intuitionistic fuzzy environment, i.e. to maximize the degree of acceptance of IF objectives and constraints and to minimize the degree of rejection of IF objectives and constraints, the following needs to be solved:

Maximize

Minimize $\quad v_{\mathrm{i}}(\mathrm{x}), \mathrm{i}=1 \ldots(\mathrm{p}+\mathrm{q})$,

Subject to the constraints

$$
\begin{array}{ll}
v_{i}(x) \geq 0, & i=1 \ldots(p+q), \\
\mu_{i}(x) \geq v_{i}(x), & i=1 \ldots(p+q), \\
\mu_{i}(x)+v_{i}(x) \leq 1, & i=1 \ldots(p+q) .
\end{array}
$$

Where $\mu_{i}(x)$ denotes the degree of membership of $x$ to the $i^{\text {th }}$ IF set and $v_{i}(x)$ denotes the degree of rejection of $x$ to the $i^{\text {th }}$ IF set.

\subsubsection{Type I Generalized IF Optimization}

Now to solve optimization problem under generalized intuitionistic fuzzy environment [11], in case of Type I GIFS, to maximize the degree of acceptance of IF objectives and constraints and to minimize the degree of rejection of IF objectives and constraints, as well as to satisfy the concurrent condition, the following problem needs to be solved

$$
\begin{array}{ll}
\text { Maximize } & \mu_{i}(x), i=1 \ldots(p+q), \\
\text { Minimize } & v_{i}(x), i=1 \ldots(p+q),
\end{array}
$$

Subject to the constraints

$$
\begin{array}{ll}
\operatorname{Min}\left(\mu_{i}(x), v_{i}(x)\right) \leq 0.5, & i=1 \ldots(p+q), \\
\mu_{i}(x) \leq 1, & i=1 \ldots(p+q), \\
\left.v_{i}(x)\right) \leq 1, & i=1 \ldots(p+q) .
\end{array}
$$

Next, it is well known that if in an optimization problem $G$ denotes goal and $\mathrm{C}$ denotes constraints, decision $\mathrm{D}$ defined by $D=G \cap C$

$=\left\{<x, \mu_{G}(x) \cap \mu_{C}(x), v_{G}(x) \cup v_{C}(x)>: x \in R^{n}\right\}$.

This operator can be easily generalized and applied to the IFO problem:

$$
\begin{aligned}
& D=\left\{<x, \mu_{D}(x), v_{D}(x)\right)>: x \in R^{n} \text { and } \\
& \mu_{D}=\bigcap_{i=1}^{p+q} \mu_{i}, v_{D}=\bigcup_{i=1}^{p+q} v_{i}
\end{aligned}
$$

Hence $\mu_{D}=\min _{i=1}^{p+q} \mu_{i}, x \in R^{n}, \mu_{D} \leq \mu_{i} \quad$ and $\nu_{D}=\max _{i=1}^{p+q} v_{i}, x \in R^{n}, v_{D} \geq v_{i}$.

This can be transformed to the following system of equations:

$$
\begin{aligned}
& \alpha \leq \mu_{i}(x), i=1,2, \ldots,(p+q), \\
& \beta \geq v_{i}(x), i=1,2, \ldots,(p+q), \\
& \min (\alpha, \beta) \leq 0.5, \\
& \alpha \leq 1, \beta \leq 1 .
\end{aligned}
$$

Where $\alpha$ denotes the minimal acceptable degree of objective(s) and constraints and $\beta$ denotes the maximal degree of rejection of objective(s) and constraints.

Hence in the case of type I GIFS, it becomes a crisp optimization problem with single objective function as follows:

$$
\begin{aligned}
& \operatorname{Max} \quad \alpha-\beta \\
& \alpha \leq \mu_{i}(x), i=1,2, \ldots,(p+q), \\
& \beta \geq v_{i}(x), i=1,2, \ldots,(p+q), \\
& \quad \min (\alpha, \beta) \leq 0.5, \\
& \quad \alpha \leq 1, \\
& \beta \leq 1 .
\end{aligned}
$$


Now, the issue is to solve this optimization problem. It may be noted that following two cases may arise.

Case I: $\min (\alpha, \beta)=\alpha$. In that case, the problem becomes

$$
\begin{aligned}
& \operatorname{Max} \quad \alpha-\beta \\
& \alpha \leq \mu_{i}(x), i=1,2, \ldots,(p+q), \\
& \beta \geq v_{i}(x), i=1,2, \ldots,(p+q), \\
& \alpha \leq 0.5, \\
& \beta \leq 1 .
\end{aligned}
$$

It can be solved by using LINGO or any other software and the solution, if it exists, satisfies the objective with degree of acceptance less than or equal to 0.5 and with some degree of rejection.

Case II: $\min (\alpha, \beta)=\beta$. In that case, the problem becomes

$$
\begin{aligned}
& \operatorname{Max} \quad \alpha-\beta \\
& \alpha \leq \mu_{i}(x), i=1,2, \ldots,(p+q), \\
& \beta \geq v_{i}(x), i=1,2, \ldots,(p+q), \\
& \beta \leq 0.5, \\
& \alpha \leq 1 .
\end{aligned}
$$

This can be solved by using LINGO or any other software and the optimum solution, if it exists, satisfies the objective with degree of rejection less than or equal to 0.5 and with some degree of acceptance.

It is up to the decision maker to make the final call. When the target is to make the degree of rejection less than or equal to 0.5 , case II is chosen where as if the target is to make the degree of acceptance less than or equal to 0.5 , case $I$ is chosen. Otherwise both the problems be solved and the better result of crisp (initial) objective function may be taken as the optimum decision. So, the decision maker gets more flexibility in terms of choice over the optimum decision.

\subsubsection{Type II Generalized IF Optimization}

Similarly in case of type II GIFS, it becomes a crisp optimization problem with single objective function as follows

$$
\begin{aligned}
& \operatorname{Max} \quad \alpha-\beta \\
& \alpha \leq \mu_{i}(x), i=1,2, \ldots,(p+q), \\
& \beta \geq v_{i}(x), i=1,2, \ldots,(p+q), \\
& \alpha * \beta \leq 0.25, \\
& \alpha \leq 1, \\
& \beta \leq 1 .
\end{aligned}
$$

It is solved by some software tool such as LINGO etc.

\section{NUMERICAL EXAMPLE}

The same transportation problem considered by Angelov [1] in 1997 is taken for the sake of simplicity alone. It states that costs of a delivery from the $i^{\text {th }}$ port to the $j^{\text {th }}$ market (in thousands of dollars) are given as in the respective cells of Table 1.

Table 1. Delivery costs from port(s) to market(s)

\begin{tabular}{|c|c|c|c|c|c|}
\hline & $\begin{array}{c}\text { Mar } \\
\text { ket 1 }\end{array}$ & $\begin{array}{c}\text { Marke } \\
\mathbf{t ~ 2}\end{array}$ & $\begin{array}{c}\text { Marke } \\
\mathbf{t ~ 3}\end{array}$ & $\begin{array}{c}\text { Marke } \\
\mathbf{t ~ 4}\end{array}$ & $\begin{array}{c}\text { Capacit } \\
\mathbf{y}\end{array}$ \\
\hline Port 1 & 10 & 7 & 4 & 1 & 400 \\
\hline Port 2 & 2 & 7 & 10 & 6 & 150 \\
\hline Port 3 & 8 & 5 & 3 & 2 & 350 \\
\hline Demand & 200 & 200 & 100 & 350 & - \\
\hline
\end{tabular}

The demands of loads in each market and the capacity of loads at each port are given (in tons) in the last column (row) of Table 1. An optimal transportation plan $\mathrm{x}\left(\mathrm{x} \in R^{n}\right)$ that minimizes the cost has to be determined. Practically the demands of markets are determined on the basis of sales forecasting. When the prognosis for Market 4 is about 350(t), the following IF set, according to Angelov, be a more realistic description:

$$
\begin{aligned}
& \mu_{4}=\frac{1}{1+0.01\left(x_{14}+x_{24}+x_{34}-350\right)^{2}}, \\
& v_{4}=\frac{\left(x_{14}+x_{24}+x_{34}-350\right)^{2}}{500+\left(x_{14}+x_{24}+x_{34}-350\right)^{2}} .
\end{aligned}
$$

It means that the degree of rejection $\left(v_{4}\right)$ is also defined which determines the worst admissible case. In general, this is not simply a complement to the degree of acceptance. The degree of acceptance $\left(\mu_{4}\right)$ of values of the demand in Market 4 increases more rapidly than the rejection $\left(v_{4}\right)$ decreases such that their sum is less than 1. By analogy, the rest of the IF sets are defined. Three of them determine the demand of Market 1, Market 2 and Market 3 and the other three sets determine the capacity of Port 1, Port 2 and Port 3, taking into account the subjective estimation of acceptance of various values of the demand and the capacity. It is supposed that the prognosis for Market 2 is about $200(\mathrm{t})$. Thus, the membership and non membership functions are as follows

$$
\begin{aligned}
& \mu_{2}=\frac{1}{1+0.01\left(x_{12}+x_{22}+x_{32}-200\right)^{2}}, \\
& \nu_{2}=\frac{\left(x_{12}+x_{22}+x_{32}-200\right)^{2}}{500+\left(x_{12}+x_{22}+x_{32}-200\right)^{2}} .
\end{aligned}
$$

Angelov assumed that the demand of Market 1 and Market 3 and the capacity of all ports are defined by crisp sets [1]. The IF objective can be determined by degrees of acceptance $\left(\mu_{0}\right)$ and rejection $\left(v_{0}\right)$ of the cost function as follows:

$$
\begin{array}{cc}
\mu_{0}=\left\{\begin{array}{cc}
1, & \sum_{i=1}^{3} \sum_{j=1}^{4} c_{i j} x_{i j}<2000 \\
\frac{2500-\sum_{i=1}^{3} \sum_{j=1}^{4} c_{i j} x_{i j}}{500}, & 2000 \leq \sum_{i=1}^{3} \sum_{j=1}^{4} c_{i j} x_{i j} \leq 2500 \\
0, & \sum_{i=1}^{3} \sum_{j=1}^{4} c_{i j} x_{i j}>2500
\end{array}\right. \\
v_{0}=\left\{\begin{array}{cc}
\frac{\left[2500-\sum_{i=1}^{3} \sum_{j=1}^{4} c_{i j}^{4} x_{i j}\right]^{2}}{250000}, & 2200 \leq \sum_{i j}^{2} \sum_{i j}^{3} \sum_{j=1}^{4} c_{i j} x_{i j} \leq 2500 \\
1, & \sum_{i=1}^{3} \sum_{j=1}^{4} c_{i j} x_{i j}>2500
\end{array}\right.
\end{array}
$$

Where $\mathrm{C}$ is given by

$$
C=\left|\begin{array}{cccc}
10 & 7 & 4 & 1 \\
2 & 7 & 10 & 6 \\
8 & 5 & 3 & 2
\end{array}\right|
$$

It is possible that a part of constraints and objective(s) are generalized intuitionistic fuzzy while others are fuzzy or crisp. In our case, the problem becomes

Maximize $\mathrm{Z}=\alpha-\beta$ 
Subject to the constraints

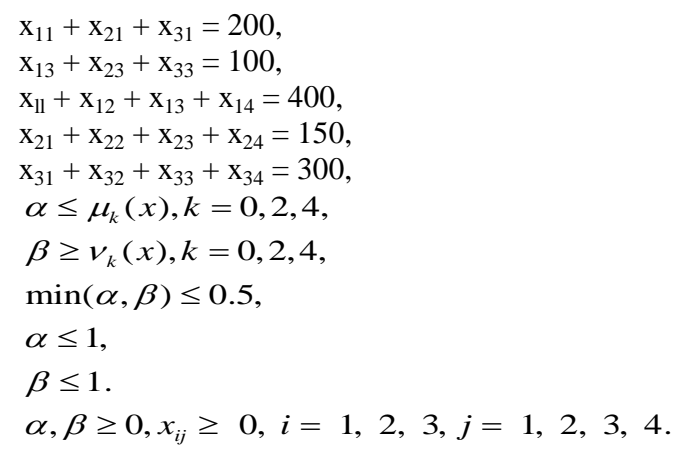

So, two cases may arise.

Case I: Here $\min (\alpha, \beta)=\alpha$. Here the problem becomes

Maximize $\mathrm{Z}=\alpha-\beta$

Subject to the constraints

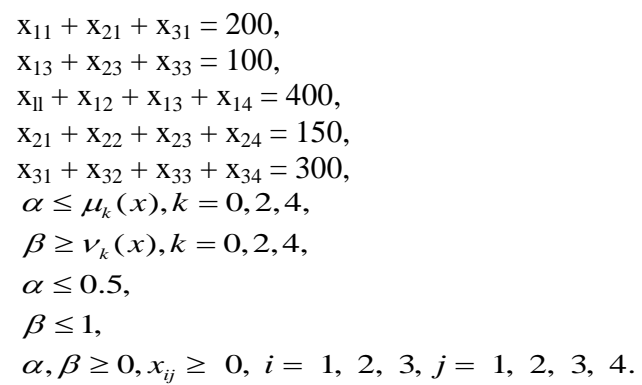

It is solved by using LINGO (Version 13.0.2.16) software and the solution satisfies the objective with degree of acceptance: 0.260 and degree of rejection: 0.068 and costs $\$ 2369.86$.

Case II: Here $\min (\alpha, \beta)=\beta$. So, the problem becomes Maximize $\mathrm{Z}=\alpha-\beta$

Subject to the constraints

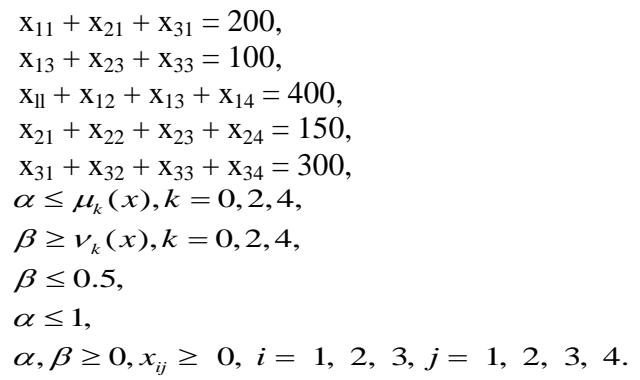

It is solved by using LINGO (Version 13.0.2.16) software and the solution satisfies the objective with degree of acceptance: 0.260 and degree of rejection: 0.068 and costs $\$ 2369.86$. It is to be noted that the optimum solution is same in both cases. Hence this is the required optimum solution.

Type II GIFS:

In this case the problem becomes

Maximize $\mathrm{Z}=\alpha-\beta$

Subject to the constraints

$$
\begin{aligned}
& x_{11}+x_{21}+x_{31}=200 \\
& x_{13}+x_{23}+x_{33}=100 \\
& x_{11}+x_{12}+x_{13}+x_{14}=400 \\
& x_{21}+x_{22}+x_{23}+x_{24}=150 \\
& x_{31}+x_{32}+x_{33}+x_{34}=300
\end{aligned}
$$

$$
\begin{aligned}
& \alpha \leq \mu_{k}(x), k=0,2,4, \\
& \beta \geq v_{k}(x), k=0,2,4, \\
& \alpha \cdot \beta \leq 0.25 \\
& \alpha \leq 1 \\
& \beta \leq 1 \\
& \alpha, \beta \geq 0, x_{i j} \geq 0, i=1,2,3, j=1,2,3,4 .
\end{aligned}
$$

It is solved by using LINGO (Version 13.0.2.16) software and the solution satisfies the objective with degree of acceptance: 0.260 and degree of rejection: 0.068 and costs $\$ 2369$.86.It is noted that the optimum solution is same as earlier for this problem.

\section{CONCLUSION}

It is to be noted that in case of generalized intuitionistic fuzzy sets, the membership and non membership function are not of complementary nature; rather their sum may be greater than 1 . It is also observed that the maximum value of sum of membership and non membership functions is two, while it is one in case of intuitionistic fuzzy set.

Here in our example, the minimum cost, level of degree of acceptance and rejection are same in all the cases. But this is not true in general. It is easy to find counter example. Even the optimum solution does not exist in all cases.

\section{REFERENCES}

[1] Angelov, P. 1997 Optimization in an intuitionistic fuzzy environment. Fuzzy Sets and Systems, Vol. 86, 299-306.

[2] Atanassov, K. T. 1986 Ideas for intuitionistic fuzzy sets equations, inequalities and optimization. Notes on Intuitionistic Fuzzy Sets, Vol. 1, No. 1, 17-24.

[3] Atanassov, K. T. 2000 Two theorems for intuitionistic fuzzy sets. Fuzzy Sets and Systems, Vol. 110, 2000, 267-269.

[4] Bellman, R. E. Zadeh, L. A. 1970 Decision making in a fuzzy environment, Management Science, Vol. 17, B141-B164.

[5] Roy, Kr. T. Banerjee, S. 2010 Solution of Single and Multi objective Stochastic Inventory Models with Fuzzy Cost Components by Intuitionistic Fuzzy Optimization Technique. Advances in Operation Research.

[6] Szmidt, E. Kacprzyk, J. 1997 Distances between intuitionistic fuzzy sets, Fuzzy Sets and Systems, Vol. $114,505-518$.

[7] Mondal, T. K. and Samanta, S. K. Generalized Intuitionistic Fuzzy Set.

[8] Zadeh, L. A. 1965 Fuzzy sets. Information and Control, $8,338-356$.

[9] Agarwala, Manish. Biswasa, Kanad K. and Hanmandlu, Madasu. 2013 Generalized intuitionistic fuzzy soft sets with applications in decision-making, Applied Soft Computing, Vol. 13, Issue 8, 3552-3566.

[10] Hanafy, I. M. Salama, A. A. Mahfouz, K. M. A. 2012 Correlation coefficients of generalized intuitionistic fuzzy sets by centroid method, IOSR Journal of Mechanical and Civil Engineering (IOSR-JMCE), Vol. 3 , Issue 5, 11-14.

[11] Sua, Zhi-xin. Xiaa, Guo-ping. Chenb Ming-yuan and Wanga. Li. 2012 Induced generalized intuitionistic fuzzy OWA operator for multi-attribute group decision making. Expert Systems with Applications, Vol. 39, Issue 2, 1902-1910. 